\title{
Triptolide induces protective autophagy and apoptosis in human cervical cancer cells by downregulating Akt/mTOR activation
}

\author{
GUANGYI QIN, PING LI and ZHUOWEI XUE \\ Department of Obstetrics and Gynaecology, Shanghai Jiao Tong University Affiliated Sixth People's Hospital, \\ Shanghai 200233, P.R. China
}

Received May 24, 2016; Accepted May 25, 2017

DOI: $10.3892 /$ ol.2018.9074

\begin{abstract}
Triptolide exhibits immunosuppressive, antiinflammatory, antifertility and antineoplastic functions. However, the anticancer effect of triptolide on cervical cancer and the underlying mechanism remains to be fully understood. The present study assessed the mechanisms underlying the effect of triptolide on the viability and apoptosis of human cervical cancer cells. SiHa cells were treated with 12.5-100.0 nM triptolide for 12, 24 or $48 \mathrm{~h}$. The present study demonstrated that triptolide inhibited viability and induced apoptosis in SiHa cells time- and dose-dependently. Furthermore, treatment with triptolide promoted autophagy and activated microtubule associated protein 1 light chain 3 $\alpha$ expression in SiHa cells. Triptolide treatment suppressed the expression of phosphorylated (p)-protein kinase B (Akt), $\mathrm{p}$-mechanistic target of rapamycin (mTOR), and p-p70S6K, activated the expression of $\mathrm{p}$-p38, mitogen-activated protein kinase (MAPK) and p53 and inhibited the expression of p-forkhead box O3 (Foxo3a) in SiHa cells. These results suggested that triptolide induces protective autophagy, suppresses cell viability and promotes apoptosis in human cervical cancer cells by inducing the autophagy-targeting phosphoinositide 3-kinase/Akt/mTOR, p38, MAPK, p53 and Foxo3a pathways.
\end{abstract}

\section{Introduction}

Cervical cancer is one of the most common malignant tumors among women globally, exhibiting high morbidity and mortality and most frequently occurring as cervical squamous cell carcinoma (1). The etiology of cervical cancer remains to be fully understood but may be associated with certain factors, including engaging in sexual intercourse with multiple

Correspondence to: Mr. Guangyi Qin, Department of Obstetrics and Gynaecology, Shanghai Jiao Tong University Affiliated Sixth People's Hospital, 600 Yishan Road, Shanghai 200233, P.R. China E-mail: qinguangyi000@sina.com

Key words: triptolide, human cervical cancer, autophagy, protein kinase $\mathrm{B}$, mechanistic target of rapamycin partners or before the age of 16, giving birth at a young age, multiple pregnancies, smoking, poor nutrition and health and infection with persistent high-risk human papilloma virus (2). Cervical cancer diagnosed at an early stage may be treated effectively and detecting and treating cervical cancer at an earlier stage is associated with an improved prognosis (3). Therefore, identifying the cells and molecular mechanisms facilitating the development of cervical cancer is a major focus for researchers globally (3).

Autophagy refers to the process of 'self-digestion' that occurs in eukaryotic cells and is characterized by the formation of an autophagosome, which possesses a double-layer membrane and wraps organelle and macromolecular proteins in the cytoplasm (4). An autophagic vacuole and a lysosome fuse to form an autophagosome, which subsequently degrades the contents inside it, thereby supplying energy and resources to support the cell metabolism and the renewal of organelles (5). Autophagy is a nonapoptotic form of eukaryotic cell death and is also known as Type-II programmed cell death (6). Autophagy is associated with numerous biological processes, including the development and growth of cells, and is a crucial biological phenomenon (7).

Apoptosis and autophagy are two causes of cell death associated with different cell morphologies (8). Apoptosis is the predominant mechanism underlying cell death, and is morphologically characterized by membrane bubbles, cell shrinkage, nuclear fragmentation, chromatin condensation, the breaking of chromosomal DNA and the formation of apoptotic bodies. Autophagic cells may be sent to the mechanistic target of rapamycin (mTOR) pathway (9). The activated mTOR kinase in mTOR complex 1 may phosphorylate, and inhibit the activity of, unc-51 like autophagy activating kinase 1 (10). mTOR complex 1 negatively regulates cell autophagy. Microtubule associated protein 1 light chain $3 \alpha$ (MAP1LC3A) and the mammalian homologous yeast protein autophagy-related protein 8 (Atg8) serve important functions in the transfer and maturity of autophagic vacuoles (10).

Protein kinase B (Akt) serves a key function in controlling the survival and apoptosis of cells, and may be activated by insulin and numerous growth factors (11). Akt functions in wortmannin-sensitive pathways with phosphoinositide 3-kinase (PI3K). Activated Akt serves numerous functions, including supporting the combination of phosphate esters, the phosphorylation and activation of Thr308 by 
pyruvate dehydrogenase kinase 1 and the phosphorylation of the C-terminal of Ser473 (8). The phosphorylation of Akt may deactivate multiple target genes, including BCL2 associated agonist of cell death, c-Raf and caspase 9, and thereby suppress apoptosis (12). Furthermore, Akt may promote the phosphorylation of mTOR, serving a key function in cell growth. Importantly, the phosphorylation of Akt may deactivate tuberin, which inhibits the activity of regulatory associated protein of MTOR complex 1 (13).

Triptolide (Fig. 1) is a type of diterpene lactone epoxide compound isolated from Tripterygium wilfordii (14). Triptolide exhibits numerous pharmacological effects, including immunosuppression, antineoplastic activity and conferring resistance to certain types of infection (15). Triptolide is used in the treatment of arthritis, autoimmune disorders, certain types of cancer, kidney disease and asthma and to suppress immune rejection following organ transplantation $(16,17)$. The present study further examined whether triptolide, a naturally occurring compound, exhibited antineoplastic activity and assessed the mechanism underlying the effect of triptolide on the growth and apoptosis of human cervical cancer cells.

\section{Materials and methods}

Cell culture. The human cervical cancer cell line SiHa was purchased from the Shanghai Cell Bank of the Chinese Academy of Sciences (Shanghai, China) and cultured in Dulbecco's modified Eagle's medium (DMEM; Hyclone; GE Healthcare Life Sciences, Logan, UT, USA) with $10 \%$ fetal bovine serum (Hyclone; GE Healthcare Life Sciences) at $37^{\circ} \mathrm{C}$ under $5 \% \mathrm{CO}_{2}$ conditions with saturated humidity.

Cell viability assay. SiHa cells were treated with 0-100 nM triptolide (Sigma-Aldrich; Merck KGaA, Darmstadt, Germany) for 12,24 or $48 \mathrm{~h}$. Cell viability was assessed using an MTT dye reduction assay. SiHa cells were seeded $\left(1 \times 10^{4}\right.$ cells $\left./ \mathrm{ml}\right)$ onto 96-well plates and incubated overnight at $37^{\circ} \mathrm{C}$. Subsequently, $40 \mu \mathrm{l}$ MTT was added onto the cells and the plates were incubated for $4 \mathrm{~h}$ at $37^{\circ} \mathrm{C}$. DMEM was then removed and dimethyl sulfoxide was added onto the cells and the plates were incubated for $20 \mathrm{~min}$ at $37^{\circ} \mathrm{C}$. Optical density was measured using an ELISA reader (Apollo LB 9110; Berthold Technologies GmbH \& Co. KG, Bad Wildbad, Germany) at $490 \mathrm{~nm}$. This experiment was repeated three times.

Immunofluorescence of autophagy staining. SiHa cells were incubated with triptolide $(0,12.5,25$ and $50 \mathrm{nM})$ for $48 \mathrm{~h}$ at $37^{\circ} \mathrm{C}$ and washed with PBS, fixed with $75 \%$ ethanol on ice for $30 \mathrm{~min}$. SiHa cells perforated with $0.25 \%$ Tris- 100 in PBS for $15 \mathrm{~min}$ and blocked with $5 \%$ bovine serum albumin in PBS for $1 \mathrm{~h}$ at $37^{\circ} \mathrm{C}$. SiHa cells were stained with MAP1LC3A antibodies (cat no. 3868; 1:500; Cell Signaling Technology, Inc., Danvers, MA, USA) at $4^{\circ} \mathrm{C}$ overnight and then incubated with Alexa Fluor ${ }^{\circledR} 488$ conjugate-anti-rabbit immunoglobulin $\mathrm{G}$ (H+L) (cat no. 4412; 1:1,000; Cell Signaling Technology, Inc.) and observed using a LSM 780 NLO confocal microscope (magnification, x40; Zeiss GmbH, Jena, Germany).

Flow cytometry. SiHa cells $\left(2.5 \times 10^{5}\right.$ cells $\left./ \mathrm{ml}\right)$ were seeded onto 6-well plates with DMEM and incubated with triptolide
$(0,12.5,25$ and $50 \mathrm{nM})$ for $48 \mathrm{~h}$ at $37^{\circ} \mathrm{C}$. Fluorescein isothiocyanate-Annexin V (BD Biosciences, Franklin Lakes, NJ, USA) was used to stain SiHa cells for 30 min according to the manufacturer's protocol. Propidium iodide (BD Biosciences) was also used to stain SiHa cells for $30 \mathrm{~min}$ according to the manufacturer's protocol. Flow cytometry (BD Biosciences) was used to analyze apoptosis and analyzed by CellQuest software version 3.1 (BD Biosciences, San Jose, CA, USA). This experiment was repeated 3 times.

Western blot analysis. SiHa cells $\left(2.5 \times 10^{5}\right.$ cells $\left./ \mathrm{ml}, \mathrm{n}=3\right)$ were seeded onto 6 -well plates and incubated with triptolide $(0,12.5$, 25 and $50 \mathrm{nM}$ ) for $48 \mathrm{~h}$ at $37^{\circ} \mathrm{C}$. The cells were subsequently washed with PBS and resuspended in radioimmunoprecipitation lysis buffer (Beyotime Institute of Biotechnology, Haimen, China) for $30 \mathrm{~min}$ on ice. Protein content was quantified using a Bradford protein assay (Beyotime Institute of Biotechnology). Protein (50 $\mu \mathrm{g} /$ lane) was separated using SDS-PAGE on a $10-12 \%$ gel and transferred to polyvinylidene fluoride membranes (EMD Millipore, Billerica, MA,USA). Membranes were blocked using 5\% nonfat dried milk dissolved in TBS for $1 \mathrm{~h}$ at $37^{\circ} \mathrm{C}$ and incubated overnight at $4^{\circ} \mathrm{C}$ with antibodies against phosphorylated (p)-Akt (cat. no. sc-293125, 1:500, Santa Cruz Biotechnology, Inc., Dallas, TX, USA), p-mTOR (cat. no. sc-293133, 1:500, Santa Cruz Biotechnology, Inc.), p-p70S6K (cat. no. 9204, 1:2,000, Cell Signaling Technology, Inc., Danvers, MA, USA), p-p38 (cat. no. sc-81621, 1:500, Santa Cruz Biotechnology, Inc.), p53 (cat. no. sc-126, 1:500, Santa Cruz Biotechnology, Inc.), p-forkhead box O3 (Foxo3a; cat. no. 5538, 1:2,000, Cell Signaling Technology, Inc.) and GAPDH (cat. no. AF0006, 1:5,000, Beyotime Institute of Biotechnology). Membranes were subsequently washed three times in TBS-Tween $(0.1 \%)$ and incubated with horseradish peroxidase-conjugated goat anti-mouse secondary antibodies (cat. no. A0216, 1:5,000, Beyotime Institute of Biotechnology) at $37^{\circ} \mathrm{C}$ for $1 \mathrm{~h}$. Membranes were subsequently visualized using a SuperSignal ${ }^{\mathrm{TM}}$ West Pico Chemiluminescent Substrate kit (Thermo Fisher Scientific, Inc., Waltham, MA, USA) and quantified using Carestream Molecular Imaging software version 5.3.4 (Carestream Health, Inc., Rochester, NY, USA). Experiments were performed in triplicate.

Statistical analysis. Data were presented as mean \pm standard error of the mean. Statistical analyses were performed using SPSS version 19.0 (IBM Corp., Armonk, NY, USA). Significant differences between the groups were determined using one-way ANOVA by Bonferroni post-hoc analysis. $\mathrm{P}<0.05$ was considered to indicate a statistically significant difference.

\section{Results}

Triptolide inhibits viability in human cervical cancer cells. The present study assessed the effect of triptolide on the viability of human cervical cancer cells using an MTT assay. Treatment with triptolide significantly inhibited the viability of SiHa cells dose- and time-dependently (Fig. 2). The viability of SiHa cells was significantly inhibited by treatment with $100 \mathrm{nM}$ triptolide for 12, 24 and $48 \mathrm{~h}$ compared with the control cells (Fig. 2). The viability of SiHa cells was significantly inhibited by $50 \mathrm{nM}$ triptolide at 24 and $48 \mathrm{~h}$ compared 


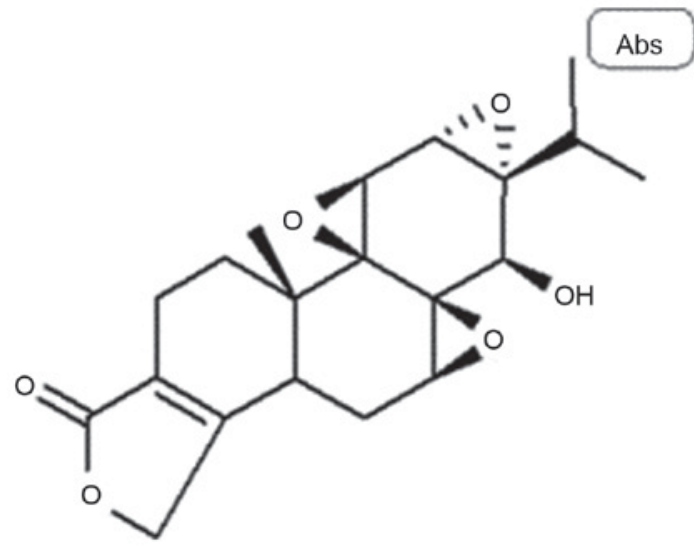

Figure 1. Chemical structure of triptolide.

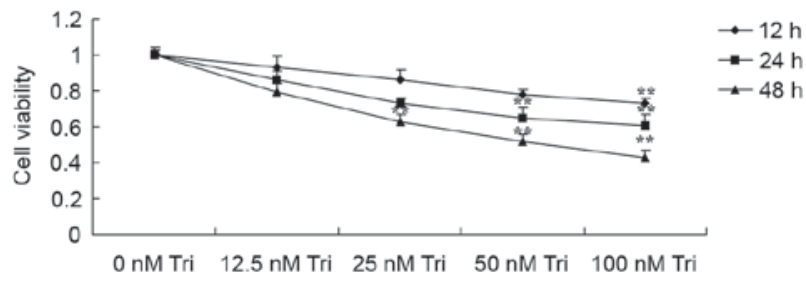

Figure 2. Tri inhibits viability in human cervical cancer cells, as assessed using an MTT assay. ${ }^{* *} \mathrm{P}<0.01$ vs. control. Tri, triptolide.

with the control cells (Fig. 2). The viability of SiHa cells was significantly inhibited by $25 \mathrm{nM}$ triptolide at $48 \mathrm{~h}$ compared with the control cells (Fig. 2).

Triptolide induces apoptosis in human cervical cancer cells. To assess the effect of triptolide on the apoptosis of human cervical cancer cells, the apoptotic rate was measured using flow cytometry. Treatment with 25 or $50 \mathrm{nM}$ triptolide significantly increased the apoptotic rate of $\mathrm{SiHa}$ cells dose-dependently, compared with the control cells (Fig. 3).

Triptolide induces protective autophagy in human cervical cancer cells. The present study examined the anticancer effect of triptolide on autophagy in human cervical cancer cells by staining and observing SiHa cells. Treatment with 25 or $50 \mathrm{nM}$ triptolide induced autophagy in SiHa cells (Fig. 4).

Triptolide regulates MAPILC3A protein expression in human cervical cancer cells. Western blot analysis demonstrated that triptolide regulated MAP1LC3A protein expression in $\mathrm{SiHa}$ cells. However, treatment with 25 or $50 \mathrm{nM}$ triptolide significantly increased MAP1LC3A protein expression in SiHa cells (Fig. 5).

Triptolide regulates $p$-Akt, $p$-mTOR and $p$-p70S6K protein expression in human cervical cancer cells. To determine whether Akt serves a function in the anticancer effect of triptolide on cervical cancer cells, western blot analysis was used to measure the protein expression of p-Akt, p-mTOR and p-p70S6K in SiHa cells. Treatment with 25 or $50 \mathrm{nM}$ triptolide significantly suppressed the expression of p-Akt, p-mTOR and p-p70S6Kprotein in SiHa cells (Fig. 6).

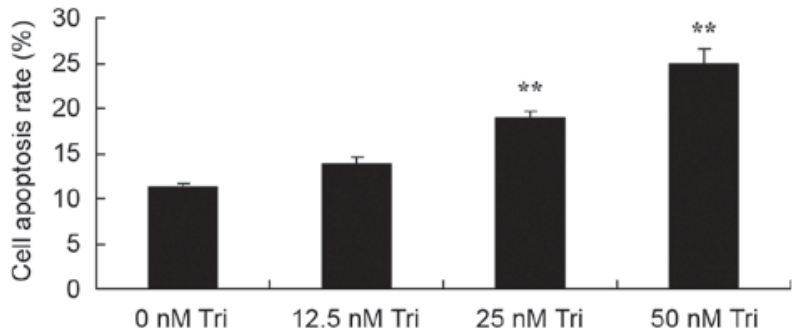

Figure 3. Tri induces apoptosis in human cervical cancer cells, as assessed by flow cytometry. ${ }^{* *} \mathrm{P}<0.01$ vs. control. Tri, triptolide.

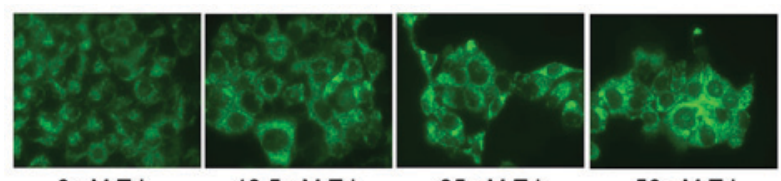

$0 \mathrm{nM}$ Tri

$12.5 \mathrm{nM}$ Tri

$25 \mathrm{nM}$ Tri

$50 \mathrm{nM}$ Tri

Figure 4. Tri induces protective autophagy in human cervical cancer cells, as assessed by confocal microscopy (magnification, x40). Tri, triptolide.

Triptolide regulates $p$-p38, p53 and p-Foxo3a protein expression in human cervical cancer cells. Expression of p-Akt, p-mTOR and p-p70S6K protein in triptolide-treated SiHa cells was detected using western blotting. Treatment with 25 or $50 \mathrm{nM}$ triptolide significantly increased p-Akt, p-mTOR and p-p70S6K protein expression in SiHa cells (Fig. 7).

\section{Discussion}

Cervical cancer is one of the most common gynecological malignant tumors globally, increasing by 500,000 cases since 2011 and responsible for 280,000 mortalities in 2012 (18). In China, 100,000 cases of cervical cancer-associated mortality are estimated annually, accounting for $\sim 1 / 3$ of the global total (19). Due to the improvement in screening for cervical cancer in medical and healthcare institutions in China, the rates of cervical cancer-associated morbidity and mortality are decreasing (20). However, young patients diagnosed with cervical cancer suffer from a relatively high mortality rate (21). The present study revealed that triptolide significantly inhibited viability and induced apoptosis in SiHa cells.

Autophagy may remove damaged organelles and proteins in cells and regulate cell growth to maintain the stability of cells and certain genes (22). However, when the expression of certain autophagy-associated proteins is altered, autophagy may be inhibited, resulting in the instability of certain genes and potentially promotion of tumor growth; at this point, active autophagy may inhibit tumor growth (23). However, tumor cells possess an increased proliferation and metabolic rate. Where tumors are not provided with sufficient blood, oxygen or nutrition, autophagy may be activated to produce recycled adenosine triphosphate, which is conducive to tumor survival (23). The results of the present study suggested that triptolide induced autophagy in SiHa cells.

Located in the anterior of autophagic vacuoles and on the surface of autophagic vacuolar membranes, MAP1LC3A is a mammalian homologue of yeast Atg8 and a common marker of the autophagic vacuolar membrane. Newly 

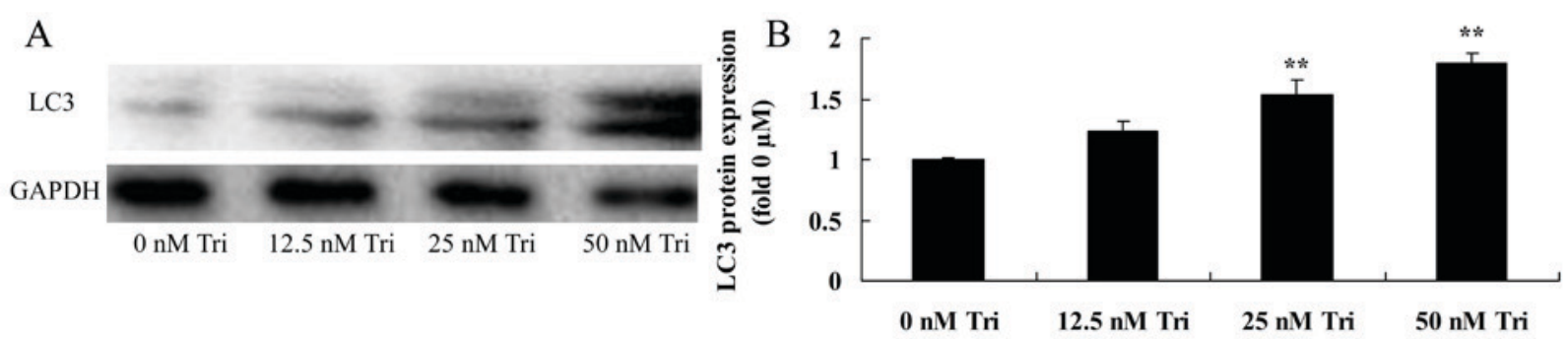

Figure 5. Tri regulates MAP1LC3A protein expression in human cervical cancer cells. (A) Western blot analysis revealed that tri regulated MAP1LC3A protein expression in human cervical cancer cells. (B) Statistical analysis of MAP1LC3A protein expression in human cervical cancer cells. ${ }^{* *} \mathrm{P}<0.01$ vs. control. Tri, triptolide; MAP1LC3A, microtubule associated protein 1 light chain $3 \alpha$.
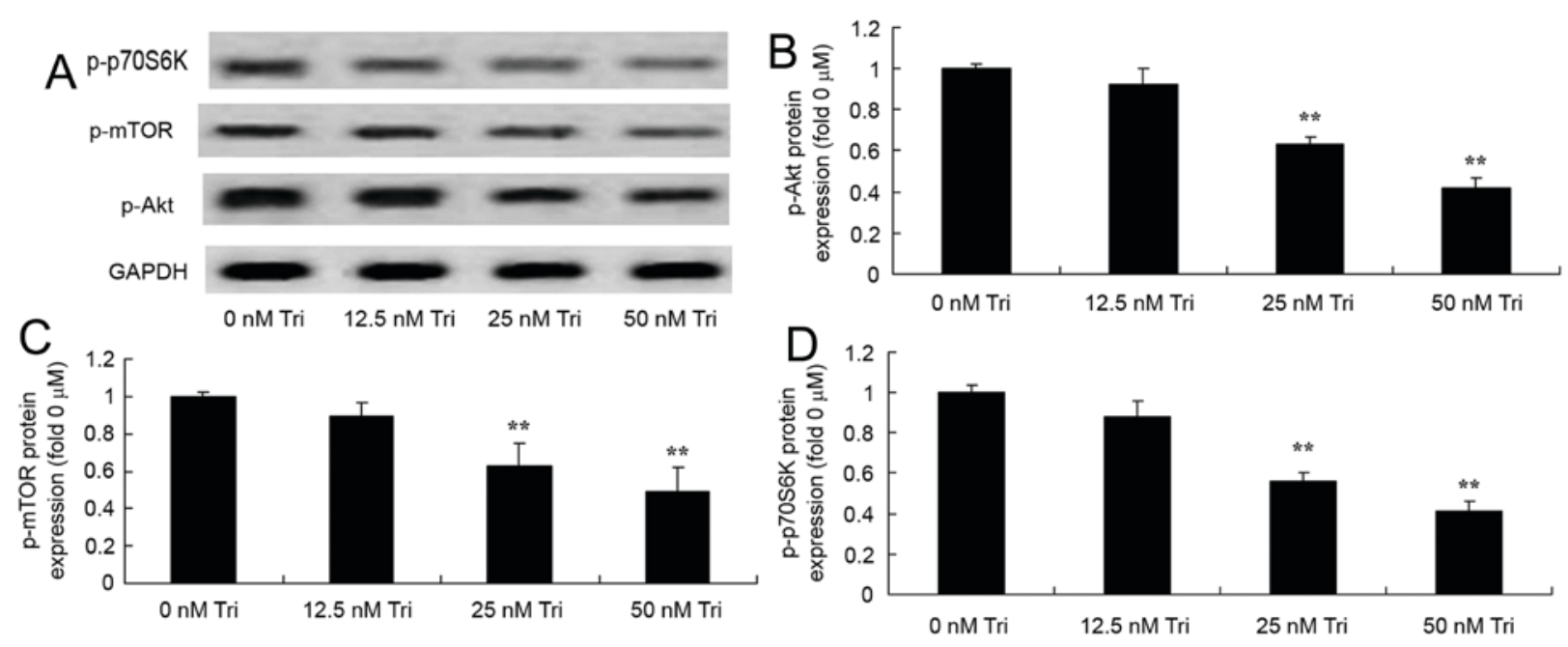

Figure 6. Tri regulates p-Akt, p-mTOR and p-p70S6K protein expression in human cervical cancer cells. (A) Western blot analysis revealed that tri regulated p-Akt, p-mTOR and p-p70S6K protein expression in human cervical cancer cells. Statistical analysis of (B) p-Akt, (C) p-mTOR and (D) p-p70S6K protein expression in human cervical cancer cells. ${ }^{* *} \mathrm{P}<0.01$ vs. control. Tri, triptolide; $\mathrm{p}$, phosphorylated; Akt, protein kinase B.
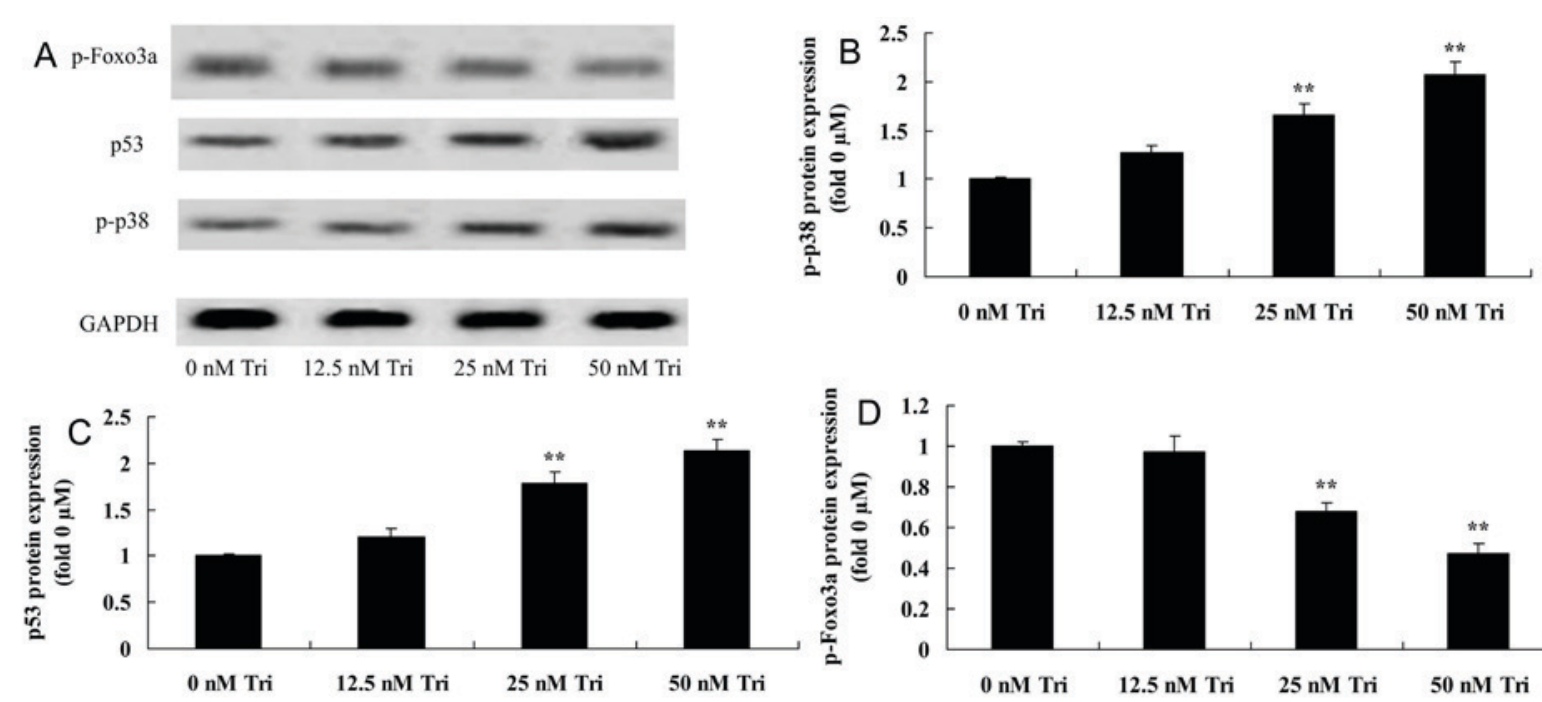

Figure 7. Tri regulates p-p38, p53 and p-Foxo3a protein expression in human cervical cancer cells. (A) Western blot analysis revealed that tri regulated p-p38, p53 and p-Foxo3a protein expression in human cervical cancer cells. Statistical analysis of (B) p-p38, (C) p53 and (D) p-Foxo3a protein expression in human cervical cancer cells. ${ }^{* *} \mathrm{P}<0.01$ vs. control. Tri, triptolide; $\mathrm{p}$, phosphorylated.

synthetized MAP1LC3A may form soluble MAP1LC3A-I following processing (24). MAP1LC3A-I may be modified by ubiquitin-like modifiers to combine with phosphatidyl ethanolamine on the surface of autophagic vacuolar membrane to form MAP1LC3A-II. Since the amount of MAP1LC3A-II is directly proportional to the number of autophagic vacuoles, 
the change in MAP1LC3A-II content may partly reflect the change in autophagic activity of cells (25). The present study demonstrated that triptolide increased MAP1LC3A protein expression in SiHa cells. Mujumdar et al (26) reported that triptolide induced death in pancreatic cancer cells via autophagic pathways. Further studies are required to examine the mechanisms underlying triptolide-induced autophagy and to provide novel insight to improve the treatment of cervical cancer.

The PI3K/Akt/mTOR pathway may serve a key function in the autophagic pathway and in the pathogenesis and clinical phenotype manifestation of cervical cancer. The $\mathrm{PI} 3 \mathrm{~K} / \mathrm{Akt} / \mathrm{mTOR}$ pathway may regulate the growth and proliferation of cells, but its function in patients with cervical cancer may be inhibited due to mutations, amplification or the absence of methylation or abnormal translation of genes following reformation (13). The activation of the PI3K/Akt/mTOR pathway in patients with cervical cancer may result in lesions of increased malignancy, which are more difficult to diagnose and treat (25). Numerous selective inhibitors of PI3K/Akt/mTOR pathway regulators are being developed, but their functions are limited due to a high rate of intrinsic or acquired drug resistance (27). Tumor cells may induce autophagy by inhibiting the activation of Akt to induce Foxo3a to translocate to the nucleus and increase autophagy-associated MAP1LC3A expression (27). In the present study, triptolide suppressed p-Akt, p-mTOR and p-p70S6K expression in SiHa cells. Zhao et al (28) reported that triptolide induces protective autophagy by suppressing mTOR expression in human prostate cancer cells. Therefore, the results of the present study suggest that triptolide induced the PI3K/Akt/mTOR/p70S6K-associated pathway to induce autophagy, which exploit in order to treat cervical cancer.

The autophagy of mammals comprises six main steps: Activation, nucleation, prolonging, closing, maturity and degeneration or dying (29). The mTOR pathway is one autophagy-associated pathway but numerous other signaling pathways and translations may regulate the autophagy of cells (30). Depending on its subcellular location, p53 may activate or inhibit autophagy (31). Autophagy may also be regulated by certain proteins, including AMP-activated proteases, Akt, p38, mitogen-activated protein kinase/extracellular signal-regulating enzymes and protease C (32). The results of the present study indicated that triptolide increased p-p38 and $\mathrm{p} 53$ protein expression and suppressed Foxo3a protein expression in SiHa cells. Xiong et al (33) suggested that triptolide exhibits an anticancer effect in human breast cancer by downregulating activated Akt and upregulating p53 expression. Park et al (34) demonstrated that triptolide inhibited the growth of THP-1 cells by inducing apoptosis through the mitogen-activated protein kinase pathway. Therefore, p38, p53 and Foxo3a may be pivotal targets in the anticancer effect of triptolide on cervical cancer.

To conclude, the results from the present study demonstrated that triptolide inhibited viability and increased the apoptosis rate in $\mathrm{SiHa}$ cells by targeting the autophagy-inducing PI3K/Akt/mTOR, p38, p53 and Foxo3a pathways. The present study further examined the PI3K/Akt/mTOR pathway and provided novel insight into the mechanism by which triptolide may function as an anticancer agent for cervical cancer therapy.

\section{Acknowledgements}

Not applicable.

\section{Funding}

No funding was received.

\section{Availability of data and materials}

The analyzed data sets generated during the study are available from the corresponding author on reasonable request.

\section{Authors' contributions}

GQ designed the experiment; PL and ZX performed the experiment. GQ analyzed the data and wrote the manuscript.

\section{Ethics approval and consent to participate}

Not applicable.

\section{Consent for publication}

Not applicable.

\section{Competing interests}

The authors declare that they have no competing interests.

\section{References}

1. Li JM, Shao JL, Zeng WJ and Liang RB: General/epidural anesthesia in combination preserves NK cell activity and affects cytokine response in cervical carcinoma patients undergoing radical resection: A cohort prospective study. Eur J Gynaecol Oncol 36: 703-707, 2015.

2. Herrero R, Quint W,Hildesheim A, GonzalezP, Struijk L, KatkiHA, Porras C, Schiffman M, Rodriguez AC, Solomon D, et al: Reduced prevalence of oral human papillomavirus (HPV) 4 years after bivalent HPV vaccination in a randomized clinical trial in Costa Rica. PLoS One 8: e68329, 2013.

3. Frumovitz M, Querleu D, Gil-Moreno A, Morice P, Jhingran A, Munsell MF, Macapinlac HA, Leblanc E, Martinez A and Ramirez PT: Lymphadenectomy in locally advanced cervical cancer study (LiLACS): Phase III clinical trial comparing surgical with radiologic staging in patients with stages IB2-IVA cervical cancer. J Minim Invasive Gynecol 21: 3-8, 2014.

4. Xu Y, Yu H, Qin H, Kang J, Yu C, Zhong J, Su J, Li H and Sun L: Inhibition of autophagy enhances cisplatin cytotoxicity through endoplasmic reticulum stress in human cervical cancer cells. Cancer Lett 314: 232-243, 2012.

5. Chen TC, Hung YC, Lin TY, Chang HW, Chiang IP, Chen YY and Chow KC: Human papillomavirus infection and expression of ATPase family AAA domain containing 3A, a novel anti-autophagy factor, in uterine cervical cancer. Int $\mathrm{J}$ Mol Med 28: 689-696, 2011.

6. Xu L, Liu JH, Zhang J, Zhang $\mathrm{N}$ and Wang ZH: Blockade of autophagy aggravates endoplasmic reticulum stress and improves Paclitaxel cytotoxicity in human cervical cancer cells. Cancer Res Treat 47: 313-321, 2015.

7. Liu Q, Luo XY, Jiang H, Yang MH, Yuan GH, Tang Z and Wang H: Hydroxychloroquine facilitates autophagosome formation but not degradation to suppress the proliferation of cervical cancer SiHa cells. Oncol Lett 7: 1057-1062, 2014.

8. Kim A, Yim NH and Ma JY: Samsoeum, a traditional herbal medicine, elicits apoptotic and autophagic cell death by inhibiting Akt/mTOR and activating the JNK pathway in cancer cells. BMC Complement Altern Med 13: 233, 2013. 
9. Li Z, Ji X, Wang W, Liu J, Liang X, Wu H, Liu J, Eggert US, Liu Q and Zhang X: Ammonia Induces autophagy through dopamine receptor D3 and MTOR. PLoS One 11: e0153526, 2016.

10. Belzile JP, Sabalza M, Craig M, Clark E, Morello CS and Spector DH: Trehalose, an mTOR-independent inducer of autophagy, inhibits human cytomegalovirus infection in multiple cell types. J Virol 90: 1259-1277, 2015.

11. Wang X, Feng Z, Li J, Chen L and Tang W: High glucose induces autophagy of MC3T3-E1 cells via ROS-AKT-mTOR axis. Mol Cell Endocrinol 429: 62-72, 2016.

12. Lv S, Xu QY, Sun EC, Zhang JK and Wu DL: Dissection and integration of the autophagy signaling network initiated by bluetongue virus infection: Crucial candidates ERK1/2, Akt and AMPK. Sci Rep 6: 23130, 2016.

13. Park SJ, Ryu J, Kim IH, Choi YH and Nam TJ: Activation of the mTOR signaling pathway in breast cancer MCF7 cells by a peptide derived from porphyra yezoensis. Oncol Rep 33: 19-24, 2015.

14. Hu G, Gong X, Wang L, Liu M, Liu Y, Fu X, Wang W, Zhang T and Wang X: Triptolide promotes the clearance of $\alpha$-synuclein by enhancing autophagy in neuronal cells. Mol Neurobiol 54 2361-2372, 2017

15. Ziaei S and Halaby R: Immunosuppressive, anti-inflammatory and anti-cancer properties of triptolide: A mini review. Avicenna J Phytomed 6: 149-164, 2016.

16. Zhu W, Hu H, Qiu P and Yan G: Triptolide induces apoptosis in human anaplastic thyroid carcinoma cells by a p53-independent but NF-kappaB-related mechanism. Oncol Rep 22: 1397-1401, 2009.

17. Jiang XH, Wong BC, Lin MC, Zhu GH, Kung HF, Jiang SH, Yang D and Lam SK: Functional p53 is required for triptolide-induced apoptosis and AP-1 and nuclear factor-kappaB activation in gastric cancer cells. Oncogene 20: 8009-8018, 2001.

18. Verhoef VM, van Kemenade FJ, Rozendaal L, Heideman DA, Bosgraaf RP, Hesselink AT, Melchers WJ, Massuger LF, Bekkers RL, Steenbergen RD, et al: Follow-up of high-risk HPV positive women by combined cytology and bi-marker CADM1/MAL methylation analysis on cervical scrapes. Gynecol Oncol 137: 55-59, 2015.

19. Rangwala R, Chang YC, Hu J, Algazy KM, Evans TL, Fecher LA, Schuchter LM, Torigian DA, Panosian JT, Troxel AB, et al: Combined MTOR and autophagy inhibition: Phase I trial of hydroxychloroquine and temsirolimus in patients with advanced solid tumors and melanoma. Autophagy 10: 1391-1402, 2014

20. Hogdal N, Juhl C, Aadahl M and Gluud C: Early preventive exercises versus usual care does not seem to reduce trismus in patients treated with radiotherapy for cancer in the oral cavity or oropharynx: A randomised clinical trial. Acta Oncol 54: 80-87, 2015.

21. Wang J, Ou J, Guo Y, Dai T, Li X, Liu J, Xia M, Liu L and He M: TBLR 1 is a novel prognostic marker and promotes epithelial-mesenchymal transition in cervical cancer. Br J Cancer 111: $112-124,2014$
22. Wei B, Huang Q, Huang S, Mai W and Zhong X Trichosanthin-induced autophagy in gastric cancer cell MKN-45 is dependent on reactive oxygen species (ROS) and NF-kB/p53 pathway. J Pharmacol Sci 131: 77-83, 2016.

23. Leisching G, Loos B, Botha M and Engelbrecht AM: A nontoxic concentration of cisplatin induces autophagy in cervical cancer: Selective cancer cell death with autophagy inhibition as an adjuvant treatment. Int J Gynecol Cancer 25: 380-388, 2015.

24. Florey O, Gammoh N, Kim SE, Jiang X and Overholtzer M: V-ATPase and osmotic imbalances activate endolysosomal LC3 lipidation. Autophagy 11: 88-99, 2015.

25. Duan J, Yu Y, Yu Y, Li Y, Wang J, Geng W, Jiang L, Li Q, Zhou X and Sun Z: Silica nanoparticles induce autophagy and endothelial dysfunction via the PI3K/Akt/mTOR signaling pathway. Int J Nanomedicine 9: 5131-5141, 2014.

26. Mujumdar N, Mackenzie TN, Dudeja V, Chugh R, Antonoff MB, Borja-Cacho D, Sangwan V, Dawra R, Vickers SM and Saluja AK Triptolide induces cell death in pancreatic cancer cells by apoptotic and autophagic pathways. Gastroenterology 139: 598-608, 2010.

27. Yu CC, Huang HB, Hung SK, Liao HF, Lee CC, Lin HY, Li SC, Ho HC, Hung CL and Su YC: AZD2014 radiosensitizes oral squamous cell carcinoma by inhibiting AKT/mTOR axis and inducing G1/G2/M cell cycle arrest. PLoS One 11: e0151942, 2016.

28. Zhao F, Huang W, Zhang Z, Mao L, Han Y, Yan J and Lei M: Triptolide induces protective autophagy through activation of the CaMKK $\beta$-AMPK signaling pathway in prostate cancer cells. Oncotarget 7: 5366-5382, 2016.

29. Kang EB and Cho JY: Effect of treadmill exercise on PI3K/AKT/mTOR, autophagy, and Tau hyperphosphorylation in the cerebral cortex of NSE/htau23 transgenic mice. J Exerc Nutrition Biochem 19: 199-209, 2015

30. Baena M, Sanguesa G, Hutter N, Sánchez RM, Roglans N, Laguna JC and Alegret M: Fructose supplementation impairs rat liver autophagy through mTORC activation without inducing endoplasmic reticulum stress. Biochim Biophys Acta 1851: 107-116, 2015.

31. Chen L, Jiang Z, Ma H, Ning L, Chen H, Li L and Qi H: Volatile oil of acori graminei Rhizoma-induced apoptosis and autophagy are dependent on p53 status in human glioma cells. Sci Rep 6: 21148, 2016.

32. Li JP, Yang YX, Liu QL, Pan ST, He ZX, Zhang X, Yang T, Chen XW, Wang D, Qiu JX and Zhou SF: The investigational Aurora kinase A inhibitor alisertib (MLN8237) induces cell cycle G2/M arrest, apoptosis, and autophagy via p38 MAPK and $\mathrm{Akt} / \mathrm{mTOR}$ signaling pathways in human breast cancer cells. Drug Des Devel Ther 9: 1627-1652, 2015.

33. Xiong J, Su T, Qu Z, Yang Q, Wang Y, Li J and Zhou S: Triptolide has anticancer and chemosensitization effects by down-regulating Akt activation through the MDM2/REST pathway in human breast cancer. Oncotarget 7: 23933-23946, 2016.

34. Park SW and Kim YI: Triptolide induces apoptosis of PMA-treated THP-1 cells through activation of caspases, inhibition of NF-kB and activation of MAPKs. Int J Oncol 43: $1169-1175,2013$ 